\title{
Experiments of Floatable UAV for Wave Dissipating Block Inspection
}

\author{
Keisuke Watanabe \\ Dept. of Navigation and Ocean Engineering, Tokai University, \\ 3-20-1 ORido Shimizuku, Shizuoka, Shizuoka, Japan \\ E-mail: keisukejapan@gmail.com \\ Koshi Utsunomiya \\ Dept. of Navigation and Ocean Engineering, Tokai University, \\ 3-20-1 ORido Shimizuku, Shizuoka, Shizuoka, Japan \\ E-mail: bebop0911@gmail.com \\ Kazuho Mitsumura \\ Dept. of Navigation and Ocean Engineering, Tokai University, \\ 3-20-1 ORido Shimizuku, Shizuoka, Shizuoka, Japan \\ E-mail:kazuho.rc@gmail.com \\ Shun Takasaki \\ Dept. of Navigation and Ocean Engineering, Tokai University, \\ 3-20-1 ORido Shimizuku, Shizuoka, Shizuoka, Japan \\ E-mail:4bkw1105@tokai-u.ac.jp
}

\begin{abstract}
In this paper, we present our modification experience of making a UAV watertight and floatable. Then we present several experiments on taking pictures considering ocean monitoring in the sea near our campus. The watertight experiments are done from our school's pier. In these experiments, we repeated to start flying the UAV from the sea surface and fall it down into the water several times. Using this UAV, we tested several experiments to take pictures of wave dissipating blocks at MIHO Island where beach line erosion is critical and many blocks are arranged to prevent waves from washing the shore. We can see the shoreline very clearly from more than $200 \mathrm{~m}$ height as a macro image and also we can inspect the blocks looking around its stacks as well as approaching near the sea surface where waves break and wave splash washes the UAV.
\end{abstract}

Keywords: Floatable UAV, environmental monitoring, coastal erosion, offshore structure monitoring

\section{Introduction}

A UAV is an effective low cost photographing apparatus for ocean environment monitoring ${ }^{1,2}$. Taking images is essential for these environment monitoring such as coastal line monitoring where beach erosion is severe, inspection of offshore structures like wind power stations or coral reef observation etc. Usually the UAV flies high above the water surface, however, it is 
possible that the UAV happens to fall down into the water due to operator's control mistake or malfunction of itself. In addition, in some operations, it is desirable for the UAV to be able to float on the water surface to save energy consumption. In those cases, we need to make the UAV watertight and floatable.

As shown in Fig.1, while the MIHO Island in Shizuoka prefecture is designated as a World Heritage Site, rapid and massive beach erosion of the island is one of the critical issues ${ }^{3,4}$. It is said that UNESCO is warning if the wave dissipating blocks will not be removed, they will cancel the WHS designation because the blocks damage the beauty of the landscape of the coastline of the island. However, the wave dissipating blocks are essential to keep shore sand from being washed away by waves or currents. There is a very important road Route 150 that faces the shore and the most important role of these blocks is protecting the road from caving or depression due to road foundation washing away.

So it is very important for us to monitor the shoreline as well as the condition of those blocks widely viewed for some long term constantly. Using watertight UAVs are one of the possible solutions for this problem.

\section{UAV modification for watertight}

To realize the UAV monitoring system, we firstly need to examine how to make it watertight and floatable. The electrical components that are vulnerable to water are GPS module, wireless communication system, motor drivers, batteries and motors for propellers. We packed those systems inside a watertight container as shown in Fig.2.

The watertight container is made of polypropylene. We chose polypropylene because it's light and no radio wave is shielded. The lid has a silicon rubber sealer and can be attached tightly to the box itself.

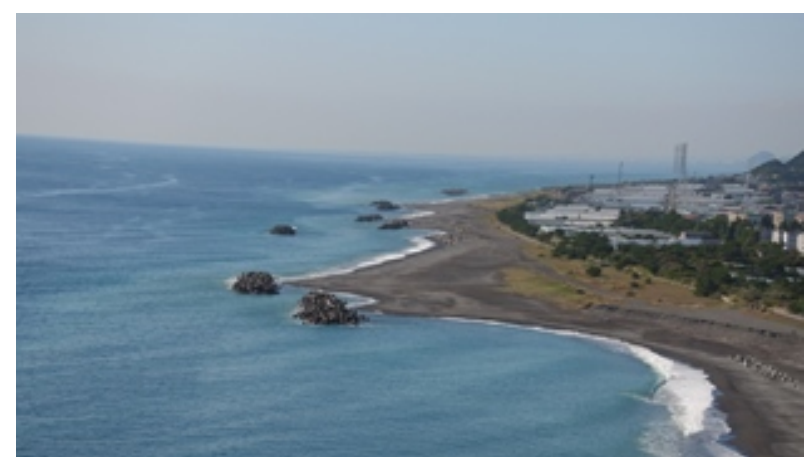

Fig.1 Coastal shore erosion and wave dissipating blocks

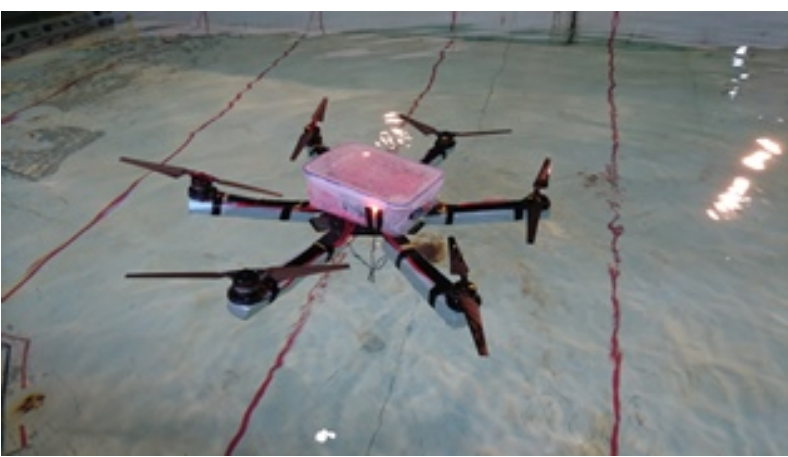

Fig.2 Electrical component in a watertight container

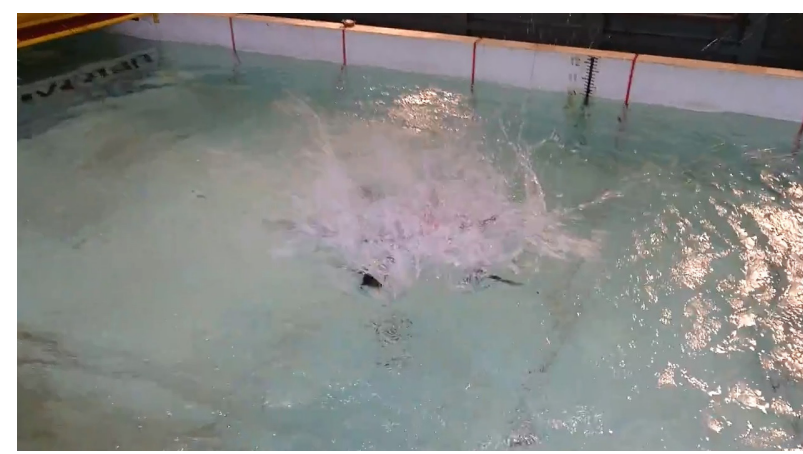

Fig.3 Diving of the UAV drone into the water

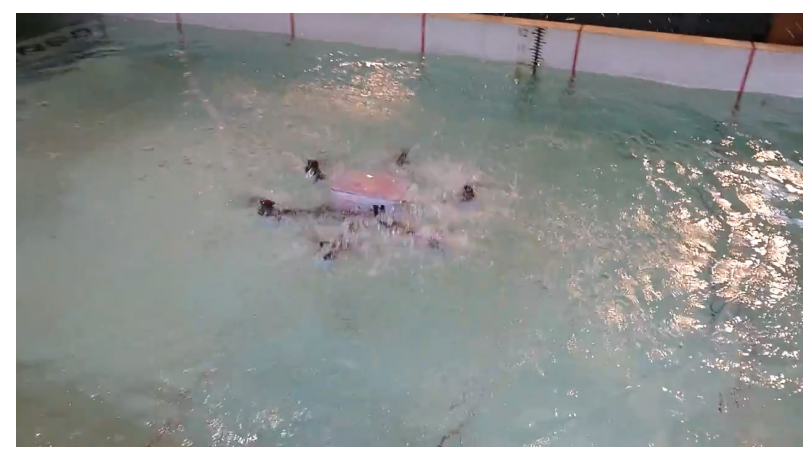

Fig.4 The UAV sunk in the tank 
We packed and arranged the electrical components inside the container and only the wires of thrusters come out of it through a hole which is sealed with silicon sealant. A float made of foamed polystyrene is attached to each arm to have enough buoyancy.

We tested it both in our tank and in the sea to check whether this method is available or not. At first, we experienced some troubles, for example, one of the wire connectors of motors inside the container is detached because of the impact of falling down to the water surface. In this case, the UAV capsized due to the flying force unbalance. In one case, the lid was fallen off due to the impact force by the water surface, so we modified the attaching apparatus using mechanical hooks to make it tighter than ever.

From Fig. 3 to Fig. 5 show an experiment of retakeoff of the UAV after diving into the water. As the float of each arm has enough buoyancy, once the UAV dives into the water, it can come back to float on the water surface immediately. Through these experiences we confirmed the UAV to be able to use on the water surface.

As the motors for the propeller are brushless motors and if we wash those motors with fresh water soon after we use them, they can be used even if we put the UAV into seawater as shown in Fig.6 and Fig.7.

\section{Monitoring of wave dissipating blocks}

After we confirmed our watertight method is available, we took several experimental pictures of the wave dissipating blocks at MIHO Island.

Fig.8 shows one area of those blocks from the height of around $100 \mathrm{~m}$. From this picture, we can see the blocks captured some sand that is put by using many dumper trucks to fill the eroded shore to recover. However, it also shows at the edge of the lined blocks, there seems another erosion started as shown in the upper left of Fig.8. We can see this situation from a wider range picture. Figure 9 shows that shore erosion proceeds in between two block areas. As we can see from the picture, the shore line vanished clearly between the two block areas. From the different colors of the water, we can see the sand flow in the sea. From the lower part of the Fig.9, we can notice a kind of vortex flow which surrounds the edge of blocks where

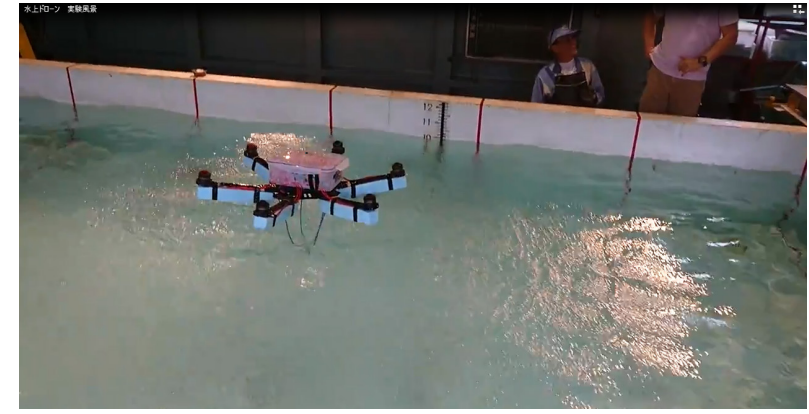

Fig.5 The UAV re-takeoff from the water

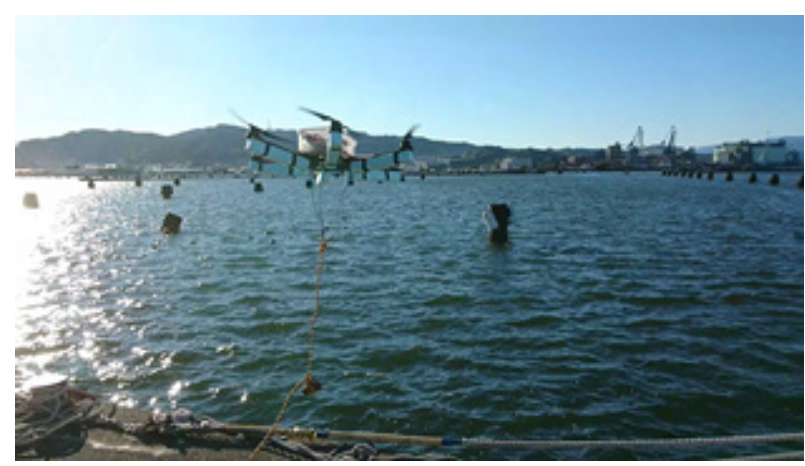

Fig.6 UAV watertight experiment in the sea

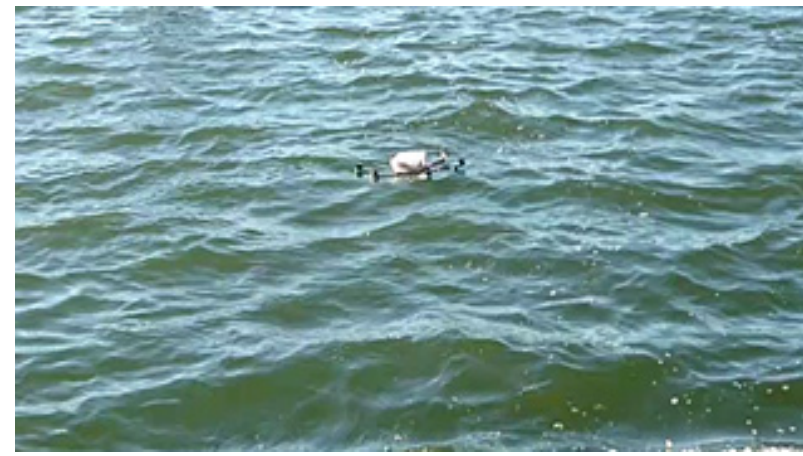

Fig.7 UAV floating experiment on the sea surface

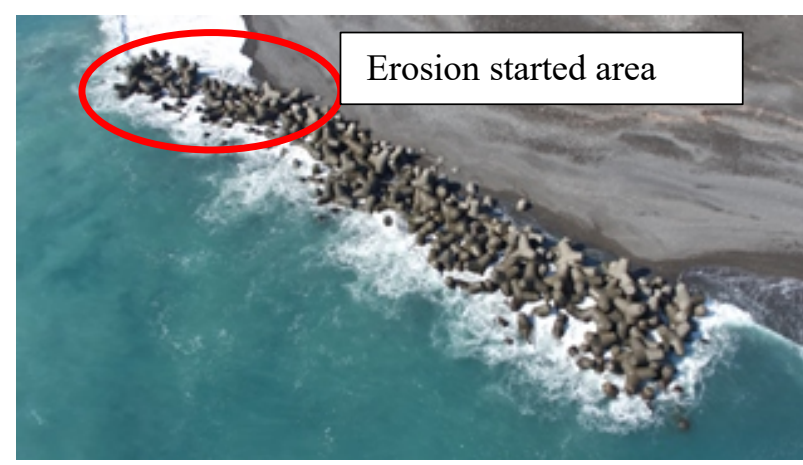

Fig.8 Sand captured by the blocks 
water color is rather green than blue compared to the left side of the picture. This implies a mountain of blocks can keep some sand around its area, however, the effect of accumulation is quite limited. For more than 20 years, hundreds of dumper trucks transported sand to fill the shore and put tons of sand into water, the shore line has not recovered yet. From the pictures taken this time, we confirmed our feeling that sand put into water never recover shoreline, instead, waves wash away sand. This picture indicates if we monitor the same point for a long term and trace changes of the landscape, we can estimate the amount of washed away sand as well as find more effective arrangements of the blocks to capture sand put by the dumper trucks every day.

Figure 10 shows the closer look of the blocks where the island of blocks are sagged. From the picture, we can find the sagging downward of the blocks. Those blocks are not old but newly put into the shore. The reason why this area is sagged downward is not clear, however, it is possible that several blocks underwater are collapsed. To find the reason, we inspected the blocks further by taking many pictures.

As shown in Fig.11, we found a broken block that looks newly put judging from its surface condition that there are few seaweeds on the surface of the block. The blocks are designed to collapse slowly absorbing the wave force to prevent sand from being washed away. However, as we can find several broken blocks like these, where the collapsing speed of a block looks too fast, and the reason of sagging downward shown in the Fig.10 seems due to this kind of collapse. We need to investigate further to find the exact reason of this kind of collapse of a block.

Furthermore, we found the evidence of wave abrasion of the block as shown in Fig.12. This is because the water around the mountain of blocks contain sand as we can see in Fig.9. From Fig.12, we guess sand mixed water abrades the block faster than we expected compared to normal sea water.

As we mentioned above, one advantage of the floating UAV is that we can observe these structures from far away to very close to them. The point where we took picture of Fig.12 is the opposite side of the shore. At this sea side of the blocks, it is very dangerous and difficult for us to approach there by a boat and keep the boat there to examine the target block because the wave is breaking and swell is strong. In addition, as there splashes salt water of waves, it is secure for us to

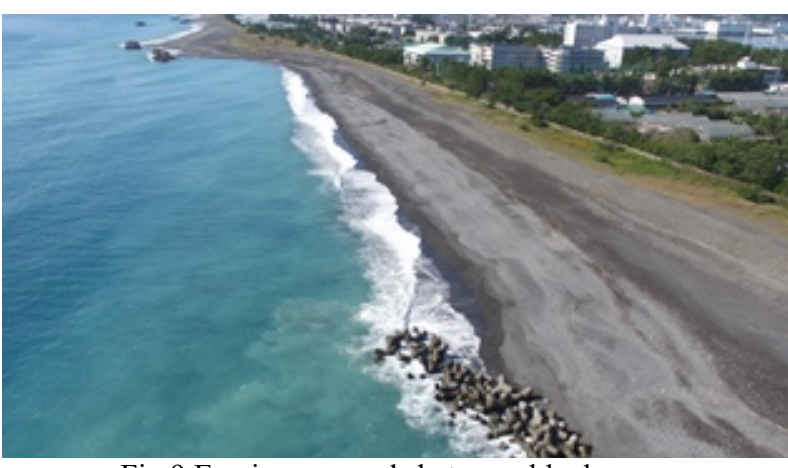

Fig.9 Erosion proceeds between block areas

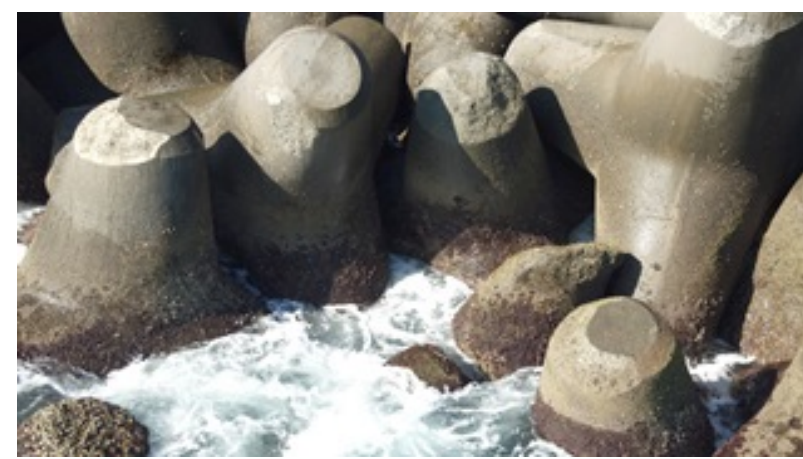

Fig.10 Downward sagging area of the blocks

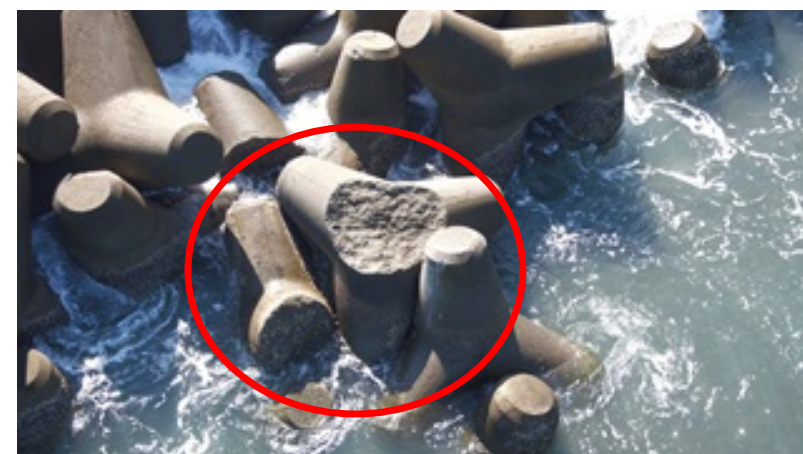

Fig.11 Broken blocks which are relatively new

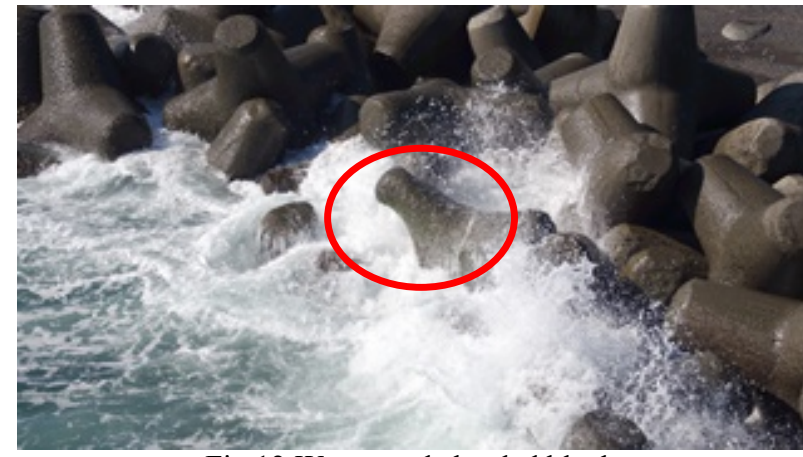

Fig.12 Wave sand abraded block 
make the UAV watertight and floatable. Thanks to this advantage we newly found the possibility that sand mixed water abrades the block faster than it is designed, comparing between the picture taken from far (Fig.9) and near (Fig.12).

\section{Estimation of lost sand amount}

The purpose of arranging wave dissipating blocks along the coast of Miho peninsula is not only breaking waves but also catching sand flowing from a big river, Abekawa river, which supplies massive sand from mountains. In addition, for more than 15 years, the government puts massive sand from shore using hundreds of trucks to fill up the shore but in vain.

As we see in the Fig.9, we cannot say the blocks worked well but massive sand had been lost. We tried to estimate the amount from the picture taken by our UAV shown in Fig. 13.

The distance from the point A to B is $117.8 \mathrm{~m}$. Using this distance, we can estimate the each side of the red triangle as $133.5 \mathrm{~m}, 471.2 \mathrm{~m}, 549.7 \mathrm{~m}$ respectively. So we can estimate the area as $27322.9 \mathrm{~m}^{2}$. The average water depth can be estimated as $3 \mathrm{~m}$, so we estimated the lost sand amount around $81968.8 \mathrm{~m}^{3}$. If we use the specific weight of sand as 2.0, then this amount equals to 163,938 tf. This means almost 16,000 10-ton trucks. Although we have to investigate more precisely how much amount of sand is losing every year, especially by measuring the water depth profile further, we have just learnt to be able to start this kind of estimation using our UAV.

\section{Conclusion}

In this paper, we modified the UAV to be able to use on the sea. We packed the electrical components into a watertight container and checked the brushless motors of propeller can be used with no special fabrication if we maintenance them correctly. Using this watertight and floatable UAV, we tried monitoring of wave dissipating blocks at MIHO shore. We found out several new things related to collapse of blocks and shore line erosion from observation using this UAV. This investigation is new because approaching the offshore side of the blocks had been difficult and expensive. Using a floating UAV, we can access such kind of dangerous zones easily with low cost. We'd like to investigate further to sophisticate this new apparatus.

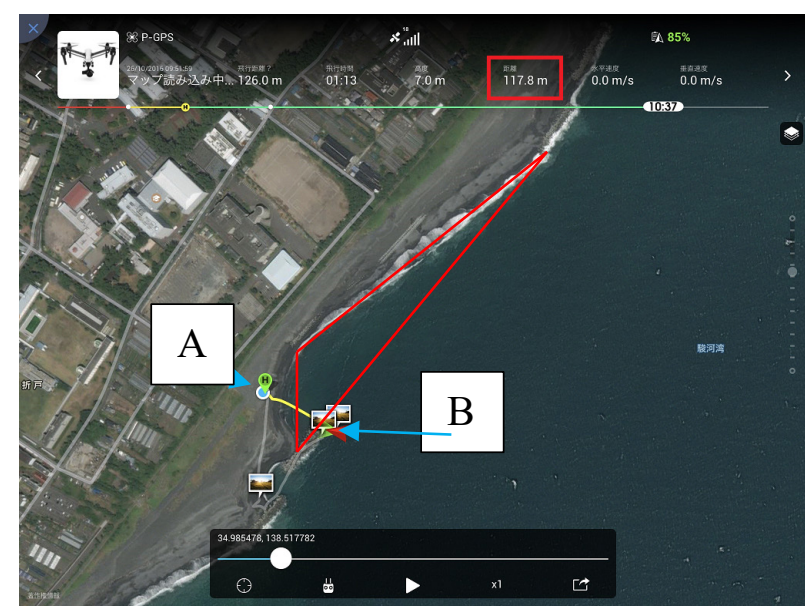

Fig.13 Lost sand estimation from the image

\section{Acknowledgement}

This research is supported by KAKANHI, Grant-in-Aid for Challenging Exploratory Research, JSPS JP26630230.

\section{References}

1. Makoto Yamada, Jun Shoji, Shun Teramoto, et al., "Exploration of submarine groundwater discharge using a drone in a coastal area of Hiji town, Oita Prefecture, Japan in summer", Journal of Japanese association of hydrological sciences, Vol.46 No.1 (2016), pp.29-38

2. Taiki Sawai, "Image Analysis of Eelgrass Field by Using Snakes Active Contour Models", Journal of Japan Society of Civil Engineers, Ser. B3 (Ocean Engineering) Vol. 73 (2017) No. 2: 827-832

3. K. Nihiguchi, S. Sato, T. Sakashita, Y. Tajima, Y. Sekiguchi, K. Tsuchihashi, "Beach Erosion Mechanism by a Structure Protruding from Shoreline and the Performance of a Sand-packed Jetty against Beach Erosion", Journal of JSCE, Vol.68, No.2 (2012)

4. K. Kimura, K. Nemoto, Y. Nagahama, O. Nishimura, "Characteristics of Beach Erosion and Effect of the Beach Erosion of the Habitat of Macrobenthos in MIHO Beach", Journal of JSCE, Vol.21, pp.229-234, (2005)

5. Takaaki Uda, Takeo Matsu-ura, Yasuhiro Osaki, Yasuhiro Ooki, Toshiro Sannami, "Erosion of OharaiIsohama Coast and Caving of Seawall of Oharai Aquarium", Journal of Japan Society of Civil Engineers, Ser. B2 (Coastal Engineering) Vol. 72 (2016) No. 2: 643648

6. Ryohei Murai, Sawahiro Shimada, Ayako Sekiyama, Taichi Kaizawa, "Monitoring of Rice Growth Status Using Drone Aerial Photography", Journal of the remote sensing society of Japan, Vol.36 (2016), pp.117-121 